\title{
Negative pressure wound therapy as a „bridge” for the final reconstruction of tissue defect following a degloving injury of the forearm - a case report
}

Izabela Ratnicka, Karolina Kondej, Justyna Jończyk, Magdalena Graczyk, Barbara Chrzanowska, Jerzy Jankau

\section{CASE REPORT}

\begin{abstract}
Degloving injuries are traumatic avulsions usually resulting in large areas of soft tissue defects. The injury involves separation of skin and subcutaneous tissue from fascia and muscles. The complex nature of these injuries requires a patientoriented multidisciplinary surgical approach and a wide variety of therapeutic options. Negative pressure wound therapy can be of benefit at every stage of the long-lasting treatment.

In this paper, we present a case of a 29-year-old male after an occupational accident with a conveyor belt leading to a degloving injury of the right forearm treated in the Department of Plastic Surgery of the Medical University in Gdańsk.

The use of NPWT, hyperbaric oxygen therapy, dermal regeneration templates, skin grafting, and early rehabilitation resulted in an excellent functional and aesthetic outcome.
\end{abstract}

Keywords-degloving injury, negative pressure wound therapy, dermal matrix,

\section{INTRODUCTION}

D EGLOVING injuries are a form of traumatic avulsions of the soft tissue, in which an extensive portion of skin and subcutaneous tissue are detached from underlying structures. 1 Shearing tangential forces applied against the body disrupt the perforating vessels at the fascial level, which eventually leads to skin necrosis. Separation of skin and subcutaneous tissues from underlying muscle and fascia leads to the creation of a space allowing for the accumulation of fluid. 2

Usually, degloving injuries are associated with a motor vehicle or occupational accidents, and most often involve the lower limbs, however, they may also occur to the trunk, scalp and the upper extremities ${ }^{1]}$ Degloving soft tissue injuries can be divided into 4 groups (patterns) reflecting the extent of tissue damage - according to the Arnez classification: 3

1) Limited degloving with abrasion/avulsion,

2) Non-circumferential degloving,

3) Circumferential single plane degloving

4) Circumferential multiplane degloving (most severe) 3

Delayed management of degloving injuries often results in full-thickness necrosis, wound infection or even necrotizing

Manuscript received 17.07.2019; revised 10.10.2019. This work did not receive any financial support.

Author affiliations: Department of Plastic Surgery, Medical University of Gdańsk , (IR, KK, JJ, MG, BC, JJ)

*Correspondence to: Izabela Ratnicka: izabelaratnicka@gmail.com

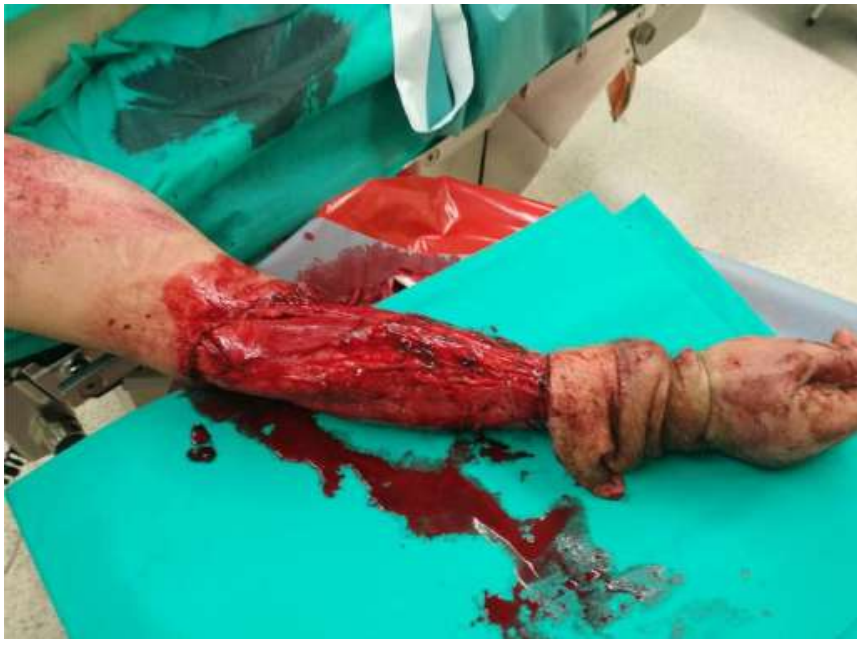

Figure 1. Male was brought to the emergency department of the University Clinical Center in Gdansk by EMTs due to injuries of the right forearm

fasciitis $[5]$ Although many methods have been defined to reform tissue integrity; defatting and primary readaptation of the avulsed flap is one of the most effective, depending on the injury pattern! 2 Negative pressure wound therapy is a method which can be helpful during every step of the surgical treatment of the wound It enhances reconstruction and improves graft success.

\section{CASE REPORT}

A 29-year-old male was brought to the emergency department of the University Clinical Center in Gdansk by EMTs due to injuries of the right forearm which he suffered as a result of an occupational accident with a conveyor belt. Clinical examination showed extensive damage to the soft tissues of the forearm from the level of the elbow joint to the wrist with exposure of the muscles and deeper forearm structures, qualified as pattern 3 of the Arnez classification (Fig. 11. In the emergency department, the fracture of bone structures was excluded using X-ray imaging, a Doppler ultrasound showed no injuries to main neurovascular bundles. The patient was qualified for surgery in an emergency setting. During the initial procedure, a thorough wound debridement and cleansing were performed and a skin-subcutaneous flap 


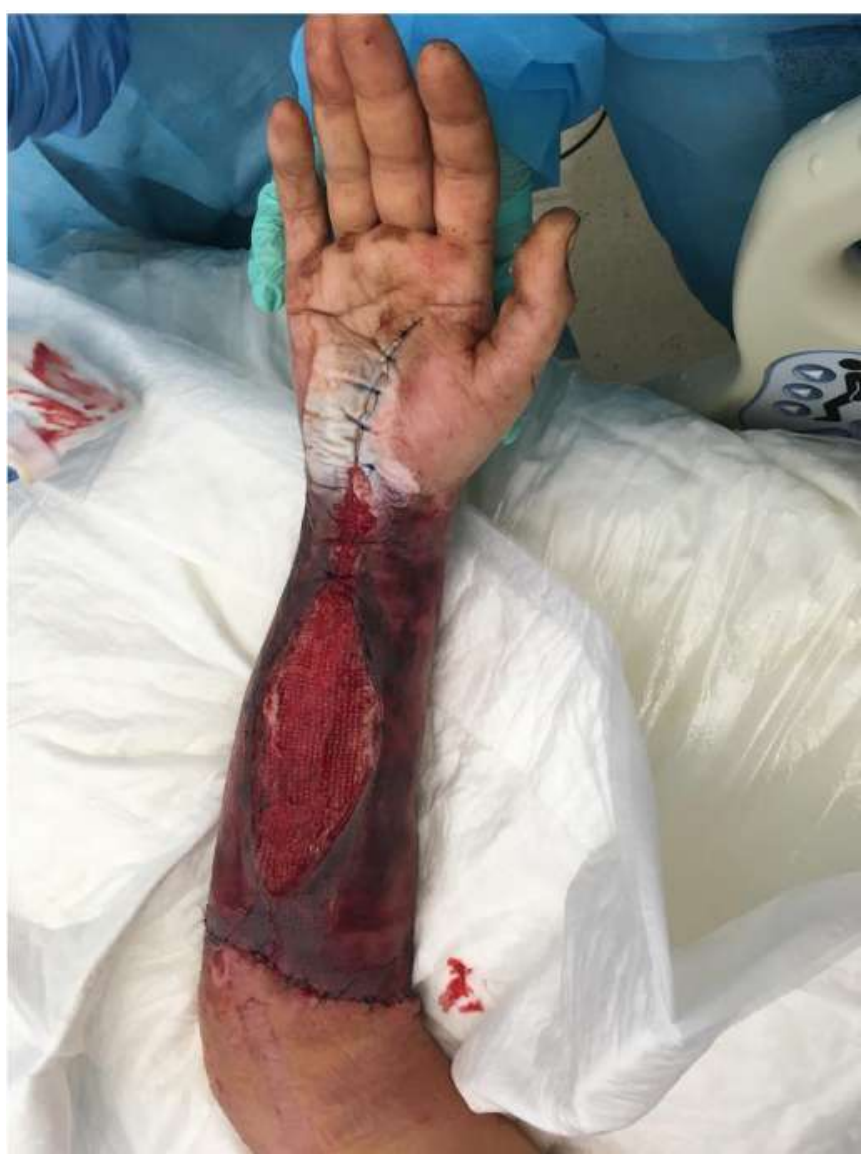

Figure 2. A negative pressure dressing with the pressure of $-100 \mathrm{mmHg}$ was applied on the incision site to reduce edema and remove exudate

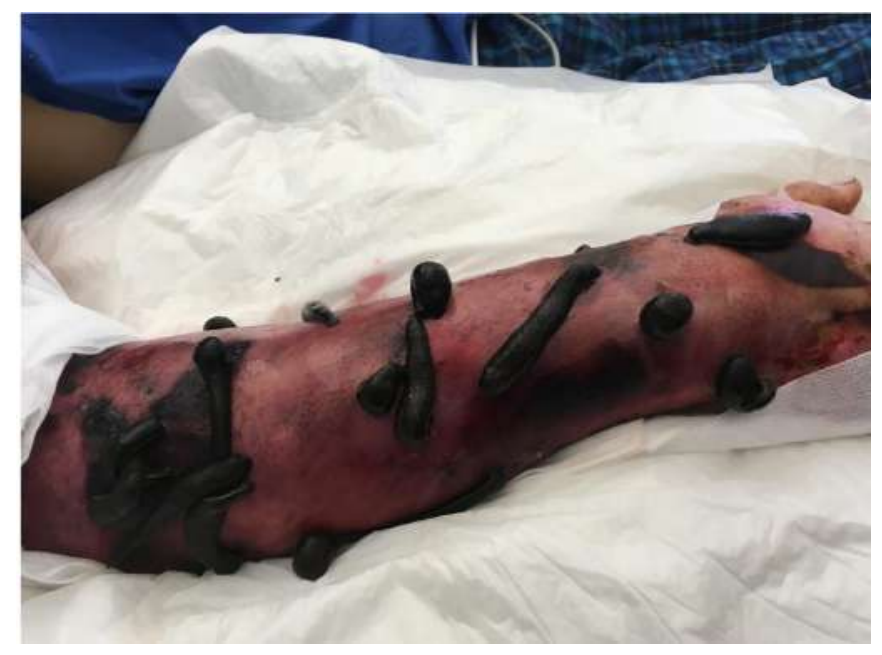

Figure 3. The last resort treatment with medical leeches

was sutured in place with a standard wound drain between the flap and the fascia. On the first postoperative day due to the growing edema in the forearm, the loss of sensation of touch in the fingers and decreased blood flow indicating the development of compartment syndrome, the patient was qualified for revision surgery. During the procedure, a longitudinal incision was made within the sutured flap, the structures of the carpal canal were released and a negative pressure

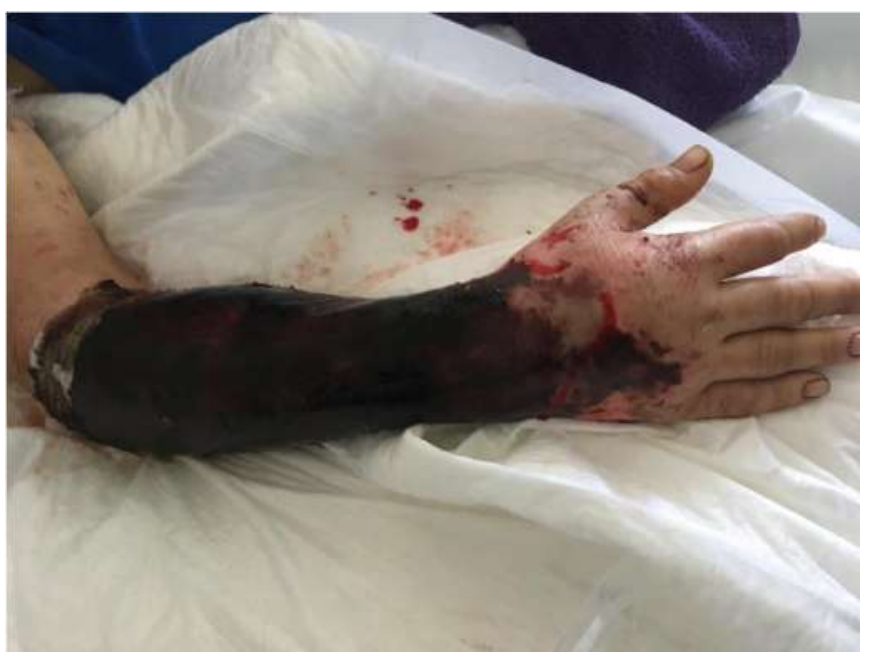

Figure 4. An increase of inflammatory parameters and deterioration of the patient's general condition was noticed

dressing with the pressure of $-100 \mathrm{mmHg}$ was applied on the incision site to reduce edema and remove exudate (Fig. 2). The patient required two exchanges of the vacuum dressing, in 4 day intervals, with intermittent negative pressure of $125 \mathrm{mmHg}$ for 7 minutes followed by 3 minutes of -75 $\mathrm{mmHg}$. At the same time, hyperbaric oxygen therapy was conducted, to improve tissue oxygenation, reduce edema and bacterial infection due to the bacteriostatic effect of hyperbaric oxygen.

Due to the features of venous stasis within the flap, a decision was made to introduce the last resort treatment with medical leeches (Fig. 3). In spite of attempts to salvage the flap, necrosis of full thickness of the skin was observed during an elective dressing change. Simultaneously, an increase of inflammatory parameters and deterioration of the patient's general condition was noticed (Fig. 4). The patient was qualified for another surgery. During the procedure, necrotic tissues extending to the level of the middle of the palmar side of the metacarpus were excised with simultaneous implantation of a dermal regeneration template (Integra) (Fig. 5). In order to fix the matrix in place, a vacuum dressing was used, which was maintained for 7 days at continuous pressure of $-90 \mathrm{mmHg}$. In the next stage of treatment, the outer silicone layer of the matrix was removed and the wound surface was covered with split-thickness skin grafts, harvested from the patients thigh, which were also fixed to the base of the wound with a vacuum dressing, through a contact layer dressing, at a constant pressure of $-90 \mathrm{mmHg}$ (Fig. 6).

On the seventh postoperative day, the NPWT was removed and complete adhesion and proper healing of skin grafts were observed throughout the transplanted area. During the hospital stay the patient received intravenous broad-spectrum antibiotic therapy based on microbiological culture results of samples acquired during the first NPWT exchange and before Interga application (Enterobacter sp., Enterococcus faecium), oral nutritional supplements and required multiple blood transfusions. The patient also started early rehabilitation which resulted in excellent mobility of the involved 


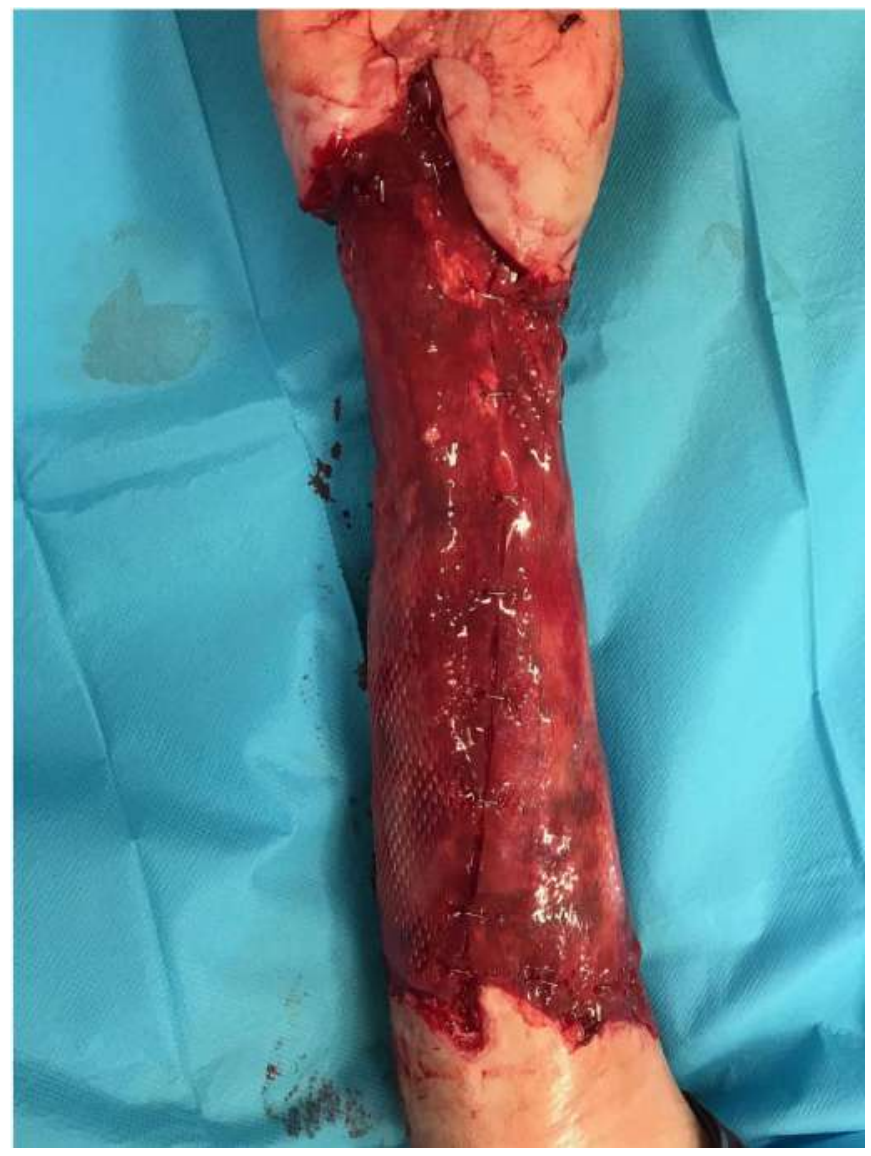

Figure 5. Implantation of a dermal regeneration template (Integra)

limb. After a forty-day postoperative period, the patient was discharged from the hospital for further treatment in an outpatient setting (Fig. 7).

\section{Discussion}

Degloving injuries, especially ones involving forearms and hands, are always a challenge in reconstructive surgery due to the extensive skin loss with the unveiling of important structures such as vessels, nerves and tendons, which must be covered with full-thickness tissue ${ }^{1}$ The outcome of the treatment should result in acceptable function and appearance. Patients with such injuries should be treated in reference centers with the possibility of microsurgical treatment. The standard involves thorough cleansing of the wound and an attempt to microsurgically attach the lobe which may not always be performed due to the patient's severe general condition, severe tissue destruction or the inability to safely transport the patient to a reference center. Repair of such extensive skin and soft tissue defects caused by degloving injuries requires in-depth knowledge of surgical techniques included in the ,reconstruction ladder”, which was developed by plastic surgeons as a canon of management. Operative techniques from the higher levels of the reconstruction ladder require advanced microsurgical skills and extensive experience. Unfortunately, the availability of facilities that can provide such highly specialized treatment in degloving injuries in Poland is insufficient.

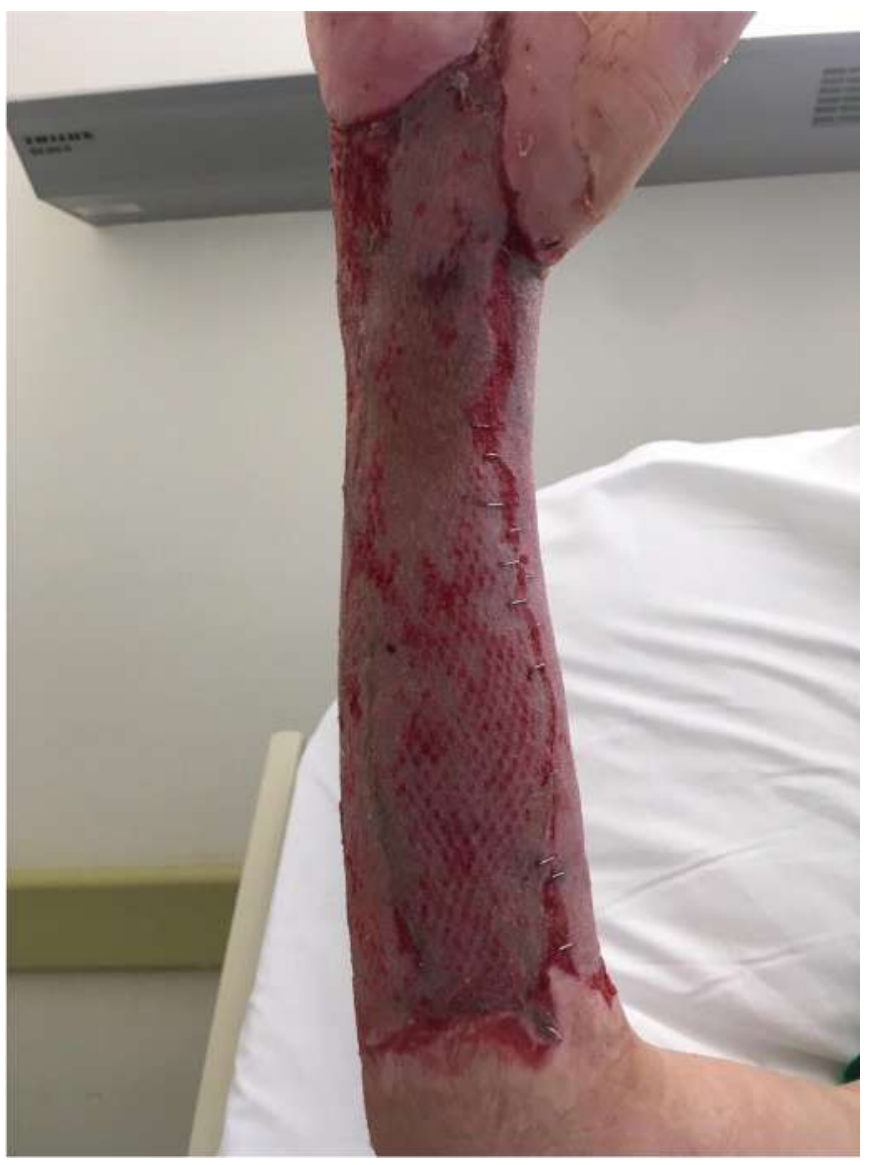

Figure 6. The outer silicone layer of the matrix was removed and the wound surface was covered with split-thickness skin grafts

When microsurgery techniques are not applicable, available literature recommends the use of detached tissues in the form of a defatted skin and subcutaneous flap and primary closure of the defect after thorough mechanical cleansing of the wound 6 In case of failure of such procedures, skin necrosis or infection, methods of secondary tissue reconstruction are used. In a situation where venous stasis is present within the flap, medical leeches may be useful. Indications and possibilities of their use, however, are limited.$^{7}$

Vacuum therapy in degloving injuries can be used at any stage of the treatment. In the case of development of compartment syndrome, the installation of a vacuum dressing allows for a reduction of tissue edema and removal of exudate. In clinical situations requiring resection of the flap due to its necrosis or infection, NPWT allows further reconstruction by stimulating granulation and preparation of the wound bed to be covered with skin grafts ${ }^{[}$When dealing with a deep loss of tissues with exposed tendons, effective in secondary reconstructions is covering them with acellular regeneration matrices (Integra), "artificial skin" or "skin substitute" 9.9 In fact, the matrices are a scaffold that facilitates the inflow of host cells and the formation of the dermis of the patient. The use of matrices for the regeneration of the dermis and covering them with autogenous transplantation of split-thickness skin grafts gives the possibility to deal with large areas of full-thickness skin defects and gives satisfying results in 


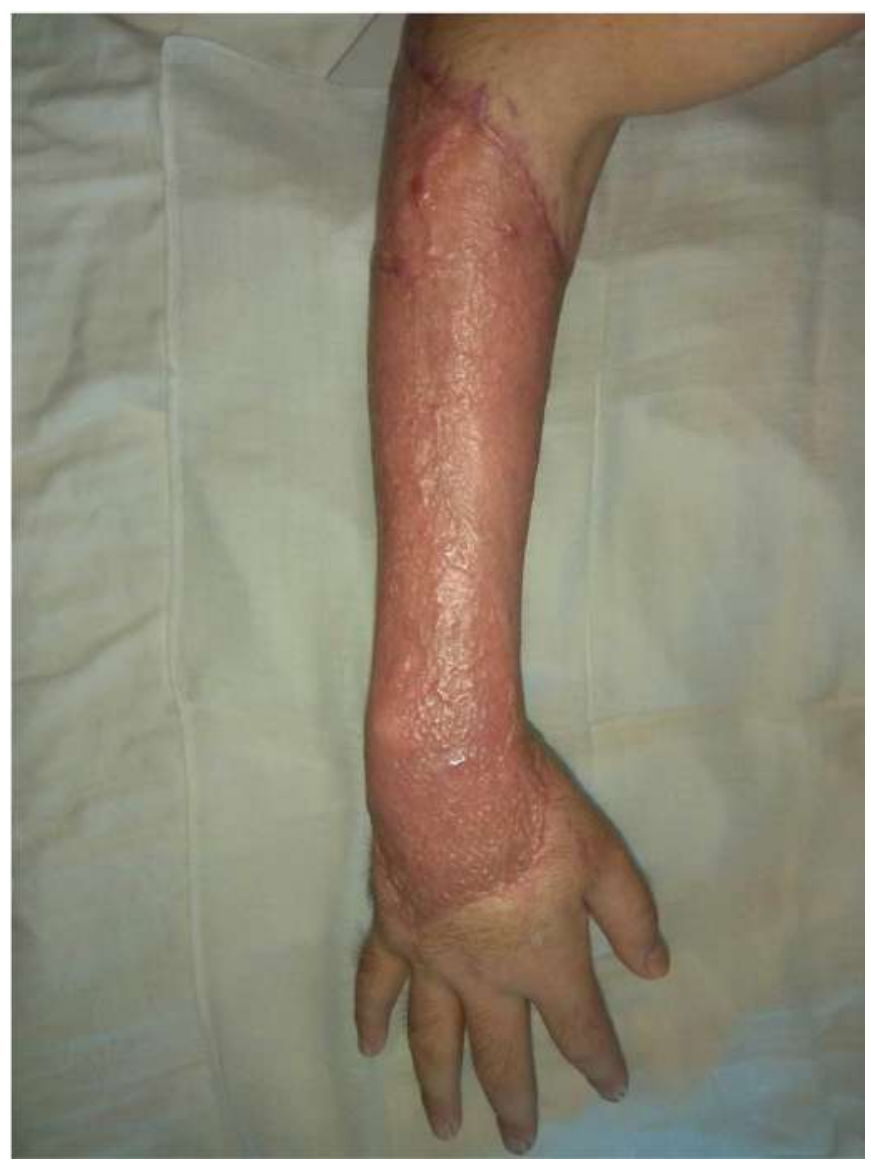

Figure 7. The patient was discharged from the hospital for further treatment in an outpatient setting

terms of aesthetics and functionality.

\section{Conclusion}

In the treatment of degloving injuries, a multidisciplinary approach is necessary and the knowledge of novel reconstruc- tive methods, negative pressure wound therapy and specialist dressings are obligatory. As in any extensive injury, early recognition and prevention of wound infection, targeted antibiotic therapy, proper nutritional support and rehabilitation are the key to the best postoperative outcome.

\section{REFERENCES}

[1] S. Hakim, K. Ahmed, A. El-Menyar, G. Jabbour, R. Peralta, S. Nabir, A. Mekkodathil, H. Abdelrahman, A. Al-Hassani, and H. Al-Thani, "Patterns and management of degloving injuries: a single national level 1 trauma center experience," World Journal of Emergency Surgery, vol. 11, no. 1, p. 35, 2016.

[2] R. Latifi, H. El-Hennawy, A. El-Menyar, R. Peralta, M. Asim, R. Consunji, and H. Al-Thani, "The therapeutic challenges of degloving softtissue injuries," Journal of emergencies, trauma, and shock, vol. 7, no. 3 , p. 228, 2014.

[3] Z. Arnez, U. Khan, and M. Tyler, "Classification of soft-tissue degloving in limb trauma," Journal of Plastic, Reconstructive E Aesthetic Surgery, vol. 63, no. 11, pp. 1865-1869, 2010.

[4] K. K. Nakarmi and S. P. Shrestha, "Degloving injury: Different ways of management," Journal of Nepalgunj Medical College, vol. 15, no. 1, pp. 54-55, 2017.

[5] P. Stabryła, J. Kulińska, A. Kasielska-Trojan, W. Gaszyński, B. Antoszewski et al., "Degloving lower leg injury-the importance of additional treatment: negative pressure and hyperbaric oxygen therapy." Polski przeglad chirurgiczny, vol. 90, no. 2, pp. 5-9, 2018.

[6] Ö. Pilancı, F. Aköz Saydam, K. Basaran, A. Datll, and E. Güven, "Management of the soft tissue extremity degloving injuries with the full-thickness grafts obtained from the avulsed flap," 2013.

[7] S. Abdullah, L. M. Dar, A. Rashid, and A. Tewari, "Hirudotherapy/leech therapy: applications and indications in surgery," Arch Clin Exp Surg, vol. 1, no. 3, pp. 172-180, 2012.

[8] M. Dini, F. Quercioli, A. Mori, G. F. Romano, A. Q. Lee, and T. Agostini, "Vacuum-assisted closure, dermal regeneration template and degloved cryopreserved skin as useful tools in subtotal degloving of the lower limb," Injury, vol. 43, no. 6, pp. 957-959, 2012.

[9] G. P. Graham, S. D. Helmer, J. M. Haan, and A. Khandelwal, "The use of integra $(\AA$ dermal regeneration template in the reconstruction of traumatic degloving injuries," Journal of Burn Care E Research, vol. 34, no. 2, pp. 261-266, 2013. 